\title{
BIBECHANA
}

A Multidisciplinary Journal of Science, Technology and Mathematics

ISSN 2091-0762 (Print), 2382-5340 (0nline)

Journal homepage: http://nepjol.info/index.php/BIBECHANA

Publisher: Research Council of Science and Technology, Biratnagar, Nepal

\section{Analytical approximate solution of higher order boundary value problems via variational iteration method}

\author{
Zobia Hamid, Jamshad Ahmad* \\ Department of Mathematics, Faculty of Science, University of Gujrat, Pakistan. \\ "Email: jamshadahmadm@gmail.com \\ Article history: Received 09 October, 2017; Accepted 31 October, 2017 \\ DOI: http://dx.doi.org/10.3126/bibechana.v15i0.18347
}

This work is licensed under the Creative Commons CC BY-NC License.

https://creativecommons.org/licenses/by-nc/4.0/

\begin{abstract}
In this paper, application of variational iteration method has been successfully extended to obtain approximate solutions of some higher order boundary value problems. We emphasize the power of the method by testing three different mathematical models of distinct orders. The results are obtained by using only little iteration.
\end{abstract}

Keywords: Nonlinear BVP; Variational iteration method; Approximate solution.

\section{Introduction}

The ordinary differential equations (ODE) with variable coefficients appear in many areas of applied sciences. Examples of these equations are Euler equation, Bessel equation and Legendre equation. Moreover, the nonlinear ordinary differential equations with variable coefficients, such as the Doffing equation, the Thomas-Fermi equation, and the Van der Pole equation, have been investigated in the literature. Linear and nonlinear ODEs with variable coefficients play a significant role in applied mathematics, physics, and engineering [1-5]. Researchers were aiming to establish reliable methods capable for solving a large class of linear or nonlinear differential and integral equations without the tangible restrictive assumptions or discretization of the variables. Recently, there has been great development of new powerful methods capable of handling linear and nonlinear equations that overcome most of the classical methods. The Adomian decomposition method, the variational iteration method, and the homotopy perturbation method are examples of the newly developed methods. The variational iteration method, now used by many researchers is capable for handling a large class of linear or nonlinear differential equations. The flexibility and adaptation provided by the method have made it readily applicable to cases where the solution is unknown in advance as is often the case in the applied sciences and engineering. The VIM provides efficient algorithm for analytic approximate solutions and numeric simulations for real-world applications in sciences [6-18]. Unlike the Adomian decomposition method, where computational algorithms are normally used to deal with the nonlinear terms, the VIM does not require the use of restrictive as-assumptions for the nonlinear terms which would complicate the analytic calculations. The VIM approaches linear and nonlinear problems directly in a like manner. The aim of this work is reconfirm the potential and applicability of the proposed method on higher order boundary value problems. 


\section{The Analysis of Variational Iteration Method}

Consider the general differential equation

$$
\mathrm{Lu}+\mathrm{Nu}=\mathrm{g}(\mathrm{x})
$$

where $L$ and $N$ are linear and nonlinear operators respectively, and $g(x)$ is the source inhomogeneous term. The variational iteration method admits the use of a correction functional for equation (1) in the form

$$
\mathrm{u}_{\mathrm{n}+1}(\mathrm{x})=\mathrm{u}_{\mathrm{n}}(\mathrm{x})+\int_{0}^{\mathrm{x}} \lambda(\mathrm{t})\left(\mathrm{Lu}_{\mathrm{n}}(\mathrm{t})+\overline{\mathrm{N}}_{\mathrm{n}}(\mathrm{t})-\mathrm{g}(\mathrm{t})\right) \mathrm{dt},
$$

where $\lambda$ is a general Lagrange's multiplier, which can be identified optimally via the variational theory, and $\overline{u_{n}}$ as a restricted variation which means $\delta \overline{u_{n}}=0$. The Lagrange multiplier $\lambda$ is crucial and critical in the method, and it can be a constant or a function. Having $\lambda$ determined, an iteration formula should be used for the determination of the successive approximations $u_{n+1}(x) ; n \geq 0$ of the solution $u(x)$. The zeroth approximation $u_{0}$ can be any selective function. However, using the initial values $u(0) ; u^{\prime}(0)$; and $u^{\prime \prime}(0)$ are preferably used for the selective zeroth approximation $u_{0}$ as will be seen later. Consequently, the solution is given by

$$
\mathrm{u}(\mathrm{x})=\operatorname{Lim}_{\mathrm{n} \rightarrow \infty} \mathrm{u}_{\mathrm{n}}(\mathrm{x})
$$

\section{Numerical Applications}

Problem 3.1 Consider a fifth order non-linear BVP

$$
u^{(v)}(x)=e^{-x} u^{(i i)}(x)
$$

with boundary conditions

$$
u(0)=u^{\prime}(0)=u^{\prime \prime}(0)=1, u(1)=u^{\prime}(1)=1 .
$$

The correctional functional is given as

$$
\mathrm{u}_{\mathrm{n}+1}(\mathrm{x})=\mathrm{u}_{\mathrm{n}}(\mathrm{x})+\int_{0}^{\mathrm{x}} \lambda\left\{\mathrm{u}_{\mathrm{n}}{ }^{(\mathrm{v})}(\mathrm{t})-\mathrm{e}^{-\mathrm{t}} \mathrm{u}_{\mathrm{n}}{ }^{(\mathrm{ii})}(\mathrm{t})\right\} \mathrm{dt},
$$

where ' $\lambda$ ' is langrage multiplier, which is identified as

$$
\lambda=\frac{(-1)^{m}(t-x)^{m-1}}{(m-1) !} .
$$

And the initial approximation is $u_{0}(x)=e^{x}$,

For $n=0$, the equation (6) gives,

$$
\begin{aligned}
\mathrm{u}_{1}(\mathrm{x}) & =\mathrm{u}_{0}(\mathrm{x})-\frac{1}{24} \int_{0}^{\mathrm{x}}(\mathrm{t}-\mathrm{x})^{4}\left\{\mathrm{u}_{0}{ }^{(5)}(\mathrm{t})-\mathrm{e}^{-\mathrm{t}} \mathrm{u}_{0}{ }^{(i i)}(\mathrm{t})\right\} \mathrm{dt}, \\
& =\mathrm{e}^{\mathrm{x}}-\frac{1}{24} \int_{0}^{\mathrm{x}}(\mathrm{t}-\mathrm{x})^{4}\left(\mathrm{e}^{\mathrm{t}}-1\right) \mathrm{dt}, \\
& =1+\mathrm{x}+\frac{\mathrm{x}^{2}}{2}+\frac{\mathrm{x}^{3}}{6}+\frac{\mathrm{x}^{4}}{24}+\frac{\mathrm{x}^{5}}{120}
\end{aligned}
$$

For $\mathrm{n}=1$,

$$
\begin{aligned}
& \mathrm{u}_{2}(\mathrm{x})=\mathrm{u}_{1}(\mathrm{x})-\frac{1}{24} \int_{0}^{\mathrm{x}}(\mathrm{t}-\mathrm{x})^{4}\left\{\mathrm{u}_{1}{ }^{(5)}(\mathrm{t})-\mathrm{e}^{-\mathrm{t}} \mathrm{u}_{1}{ }^{(i i)}(\mathrm{t})\right\} \mathrm{dt}, \\
& 57+\frac{5 \mathrm{x}^{4}}{24}-\frac{3 \mathrm{x}^{3}}{2}-34 \mathrm{x}-\frac{\mathrm{x}^{3}}{6} \mathrm{e}^{-\mathrm{x}}-3 \mathrm{x}^{2} \mathrm{e}^{-\mathrm{x}}-21 \mathrm{xe}^{-\mathrm{x}}-56 \mathrm{e}^{-\mathrm{x}}+\frac{21}{2} \mathrm{x}^{2},
\end{aligned}
$$


The approximate solution is

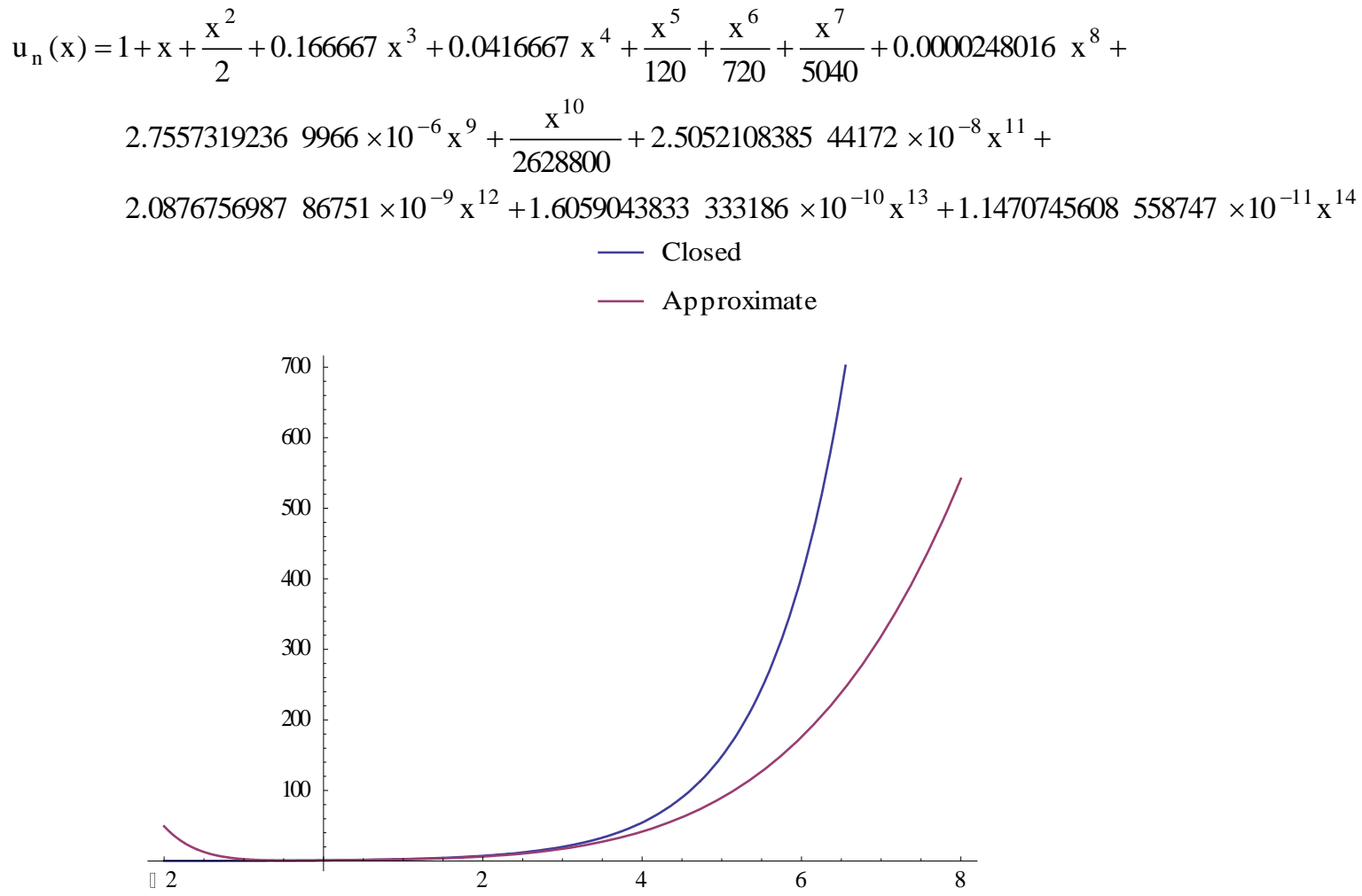

Fig 1: Comparison of exact and approximate solution.

Problem 3.2 Consider a non-linear BVP

$$
\begin{aligned}
& u^{(v i)}(x)=e^{x} u^{2}(x), \\
& u(0)=1, u^{\prime}(0)=-1, u^{\prime \prime}(0)=1, u(1)=e^{-1}, u^{\prime}(1)=-e^{-1}, u^{\prime \prime}(1)=e^{-1} .
\end{aligned}
$$

The correctional functional is given as

$$
u_{n+1}(x)=u_{n}(x)+\int_{0}^{x} \lambda\left\{u_{n}{ }^{(v)}(t)-e^{-x} u_{n}^{(i i)}(t)\right\} d t,
$$

where ' $\lambda$ ' is langrage multiplier, which is calculated by

$$
\lambda=\frac{(-1)^{m}(t-x)^{m-1}}{(m-1) \boldsymbol{l}}=\frac{(t-x)^{5}}{5 \boldsymbol{\imath}},
$$

And the initial approximation is

$$
\mathrm{u}_{0}(\mathrm{x})=\mathrm{e}^{-\mathrm{x}},
$$

For $n=0$, the equation (10) becomes,

$$
\begin{aligned}
\mathrm{u}_{1}(\mathrm{x}) & =\mathrm{u}_{0}(\mathrm{x})+\frac{1}{120} \int_{0}^{\mathrm{x}}(\mathrm{t}-\mathrm{x})^{5}\left\{\mathrm{u}_{\mathrm{o}}(\mathrm{vi})(\mathrm{t})-\mathrm{e}^{\mathrm{t}}\left(\mathrm{u}_{0}{ }^{(\mathrm{ii})}(\mathrm{t})\right)\right\} \mathrm{dt}, \\
& =\mathrm{e}^{-\mathrm{x}}+\frac{1}{120} \int_{0}^{\mathrm{x}}(\mathrm{t}-\mathrm{x})^{5}\left\{\mathrm{e}^{-\mathrm{t}}-\mathrm{e}^{\mathrm{t}}\left(\mathrm{e}^{-\mathrm{t}}\right)\right\} \mathrm{dt}, \\
& =1-\mathrm{x}+\frac{\mathrm{x}^{2}}{2 !}-\frac{\mathrm{x}^{3}}{3 \mathrm{t}}+\frac{\mathrm{x}^{4}}{4 !}-\frac{\mathrm{x}^{5}}{5 !},
\end{aligned}
$$




$$
\begin{gathered}
\mathrm{u}_{2}(\mathrm{x})=\mathrm{u}_{1}(\mathrm{x})+\frac{1}{120} \int_{0}^{\mathrm{x}}(\mathrm{t}-\mathrm{x})^{5}\left\{\mathrm{u}_{1}{ }^{(\mathrm{vi})}(\mathrm{t})-\mathrm{e}^{\mathrm{t}}\left(\mathrm{u}_{1}{ }^{(\mathrm{ii})}(\mathrm{t})\right)\right\} \mathrm{dt}, \\
=210 \mathrm{e}^{\mathrm{x}}-127 \mathrm{x}+14 \mathrm{x}^{2} \mathrm{e}^{\mathrm{x}}-\frac{7}{6} \mathrm{x}^{3} \mathrm{e}^{\mathrm{x}}+\frac{\mathrm{x}^{4} \mathrm{e}^{\mathrm{x}}}{24}-84 \mathrm{xe}^{\mathrm{x}}-\frac{69}{2} \mathrm{x}^{2}-6 \mathrm{x}^{3}-\frac{7}{12} \mathrm{x}^{4}-\frac{\mathrm{x}^{5}}{20}-209 \\
\vdots \\
\mathrm{u}(\mathrm{x})=1-\mathrm{x}+\frac{\mathrm{x}^{2}}{2}-0.166667 \mathrm{x}^{3}+0.0416667 \mathrm{x}^{4}-0.0083333 \mathrm{x}^{5}+\frac{\mathrm{x}^{6}}{720}-\frac{\mathrm{x}^{7}}{5040}+\frac{\mathrm{x}^{8}}{40320}- \\
2.755731933676053 \times 10^{-7} \mathrm{x}^{10}-2.505210866756 \times 10^{-8} \mathrm{x}^{11}+2.0876756987868175 \times 10^{-9} \mathrm{x}^{12} \\
-1.6059043836 \times 10^{-10} \mathrm{x}^{13}+1.1470745597729725 \times 10^{-11} \mathrm{x}^{14}+\cdots
\end{gathered}
$$

The closed solution of this problem is $\mathrm{e}^{-\mathrm{x}}$.

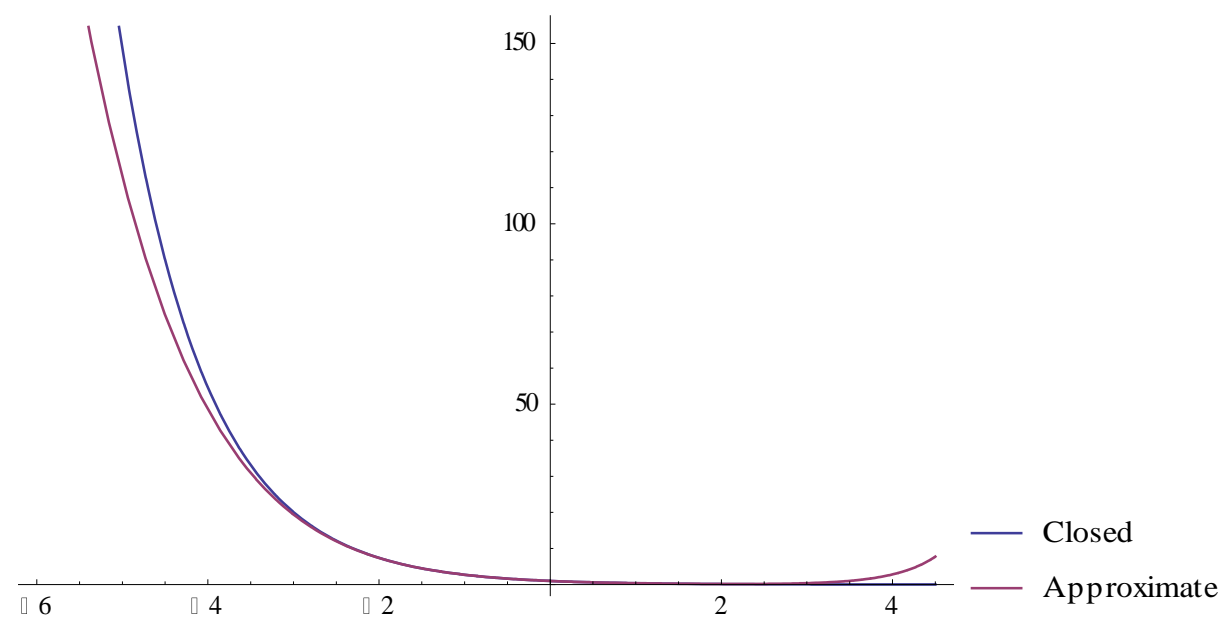

Fig.2: Comparison of closed and approximate solution.

Problem 3.3 Consider a six order non-linear BVP

$\mathrm{u}^{(\mathrm{vi})}=\mathrm{e}^{-\mathrm{x}} \mathrm{u}^{(\mathrm{ii})}(\mathrm{x})$

with boundary conditions

$$
\begin{aligned}
& \mathrm{u}(0)=\mathrm{u}^{\prime \prime}(0)=\mathrm{u}^{(\mathrm{iv})}(0)=1 \\
& \mathrm{u}(1)=\mathrm{u}^{\prime \prime}(1)=\mathrm{u}^{(\mathrm{iv})}(1)=\mathrm{e}
\end{aligned}
$$

The correctional functional is given as

$$
\mathrm{u}_{\mathrm{n}+1}(\mathrm{x})=\mathrm{u}_{\mathrm{n}}(\mathrm{x})+\int_{0}^{\mathrm{x}} \lambda\left\{\mathrm{u}_{\mathrm{n}}{ }^{(\mathrm{vi})}(\mathrm{t})-\mathrm{e}^{-\mathrm{t}} \mathrm{u}_{\mathrm{n}}{ }^{(\mathrm{ii})}(\mathrm{t})\right\} \mathrm{dt},
$$

where ' $\lambda$ ' is langrage multiplier, which is identified as

$$
\lambda=\frac{(-1)^{\mathrm{m}}(\mathrm{t}-\mathrm{x})^{\mathrm{m}-1}}{(\mathrm{~m}-1) !}=\frac{(\mathrm{t}-\mathrm{x})^{5}}{5 !}
$$

And the initial approximation is

$$
u_{0}(x)=e^{x},
$$

For $n=0$, the equation (17) becomes,

$$
\mathrm{u}_{1}(\mathrm{x})=\mathrm{e}^{\mathrm{x}}+\frac{1}{120} \int_{0}^{\mathrm{x}}(\mathrm{t}-\mathrm{x})^{5}\left\{\mathrm{e}^{\mathrm{t}}-\mathrm{e}^{-\mathrm{t}}\left(\mathrm{e}^{\mathrm{t}}\right)\right\} \mathrm{dt},
$$




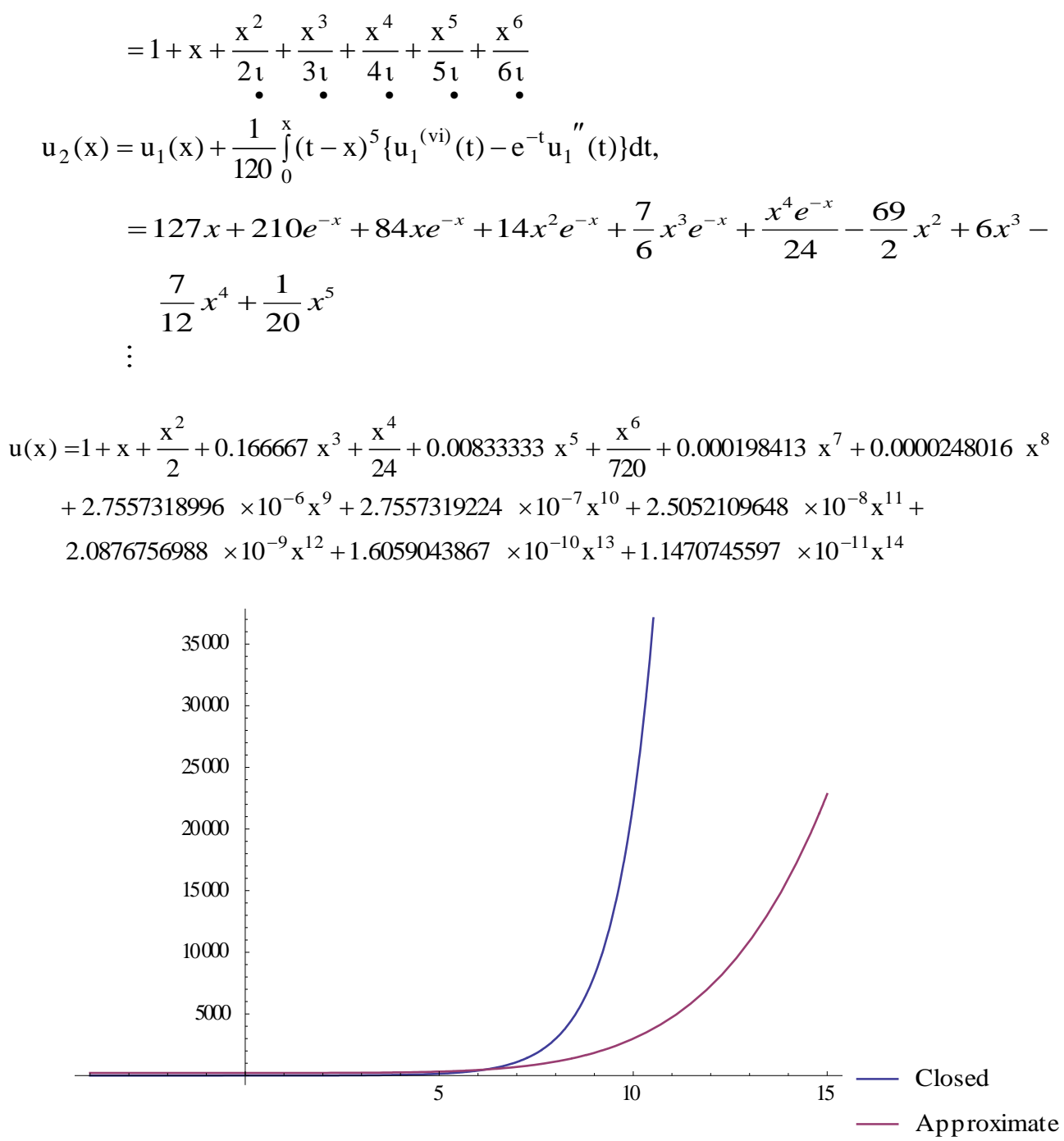

Fig.3: Comparison of exact and approximate solution.

\section{Conclusion}

In this work, the variational iteration method has been successfully employed on higher order boundary value problems by converting into corresponding system of first order differential equations. The obtained results are good agreement with the existing results in literature.

\section{References}

[1] A. M. Wazwaz, The variational iteration method for analytic treatment for linear and nonlinear ODEs, Appl. Math. Comput. 212 (2009) 120-134. doi.org/10.1016/j.amc.2009.02.003.

[2] M. Abolhasani, S. Abbasbandy, T. Allahviranloo, A New Variational Iteration Method for a Class of Fractional Convection-Diffusion Equations in Large Domains, Mathematics, 5 (2017) 26. doi.org/10.3390/math5020026.

[3] B. D. Yuliyanto, Variational iteration method for solving the population dynamics model of two species, IOP Conf. Series: J. Phys.: Conf. Ser. 795 (2017) 012044. doi.org/10.1088/1742-6596/795/1/012044. 
[4] J. H. He, Variational iteration method for autonomous ordinary differential systems, Appl. Math. Comput. 114 (2000) 115-123. doi.org/10.1016/S0096-3003(99)00104-6.

[5] J. H. He, Variational iteration method - Some recent results and new interpretations, J. Comput. Appl. Math. 207 ( 2007) 3-17. doi.org/10.1016/j.cam.2006.07.009.

[6] M. Torvattanabun, S. Koonprasert, Variational Iteration Method for Solving Eighth-Order Boundary Value Problems, Thai J Math. (2010) 121-129.

[7] A. M. Wazwaz, The variational iteration method for solving linear and nonlinear ODEs and scientific models with variable coefficient, Cent. Eur. J. Eng. 4 (2014) 64-71. doi.org/10.2478/s13531-0130141-6.uropean Journal of Engineering.

[8] M.T Akter, M.A.M Chowdhury, Variational Iteration Method for Solving Coupled Schrödinger-KleinGordon Equation, American J. Comput. Appl. Math. 7 (2017) 25-

1. doi.org/10.5923/j.ajcam.20170701.03.

[9] K. N. S. K. Viswanadham, Numerical Solution of Ninth Order Boundary Value Problems by PetrovGalerkin Method with Quintic B-splines as Basis Functions and Septic B-splines as Weight Functions, Procedia Eng. 127 (2015) 1227-1234. doi.org/10.1016/j.proeng.2015.11.470.

[10] R. P. Agarwal, Boundary Value Problems for High Ordinary Differential Equations, World Scientific, Singapore, 1986.

[11] S. T. Mohyud-Din, M.A Noor, K.I. Noor, Variation of Parameters Method for Solving a Class Of Eighth-Order Boundary-Value Problems, Int. J. Comput. Methods. 9 (2012) 1240026. doi.org/10.1142/S0219876212400269.

[12] H. N. Caglar, S. H. Caglar, E.H. Twizell, The numerical solution of fifth-order boundary value problems with sixth degree B-spline functions, Appl. Math. Lett. 12 (1999) 25-30. doi.org/10.1016/S0893-9659(99)00052-X.

[13] A. M. Wazwaz, The numerical solution of fifth-order boundary value problems by the decomposition method, J. Comput. Appl. Math. 136 (2001) 259-270. doi.org/10.1016/S0377-0427(00)00618-X.

[14] E. H. Twizell, A. Boutayeb, Numerical methods for the solution of special and general sixth-order boundary value problems, with applications to B'enard layer eigenvalue problems, Proc. R. Soc. of Lond. A, 431 (1990) 433-450. doi.org/ 10.1098/rspa.1990.0142.

[15] A. M. Wazwaz, The numerical solution of sixth-order boundary value problems by the modified decomposition method, Appl. Math. Comput. 118 (2001) 311-325. doi.org/10.1016/S00963003(99)00224-6.

[17] I. Ullah, H. Khan, M.T. Rahim, Numerical solutions of higher order nonlinear boundary value problems by new iterative method, Appl. Math. Sci. 7 (2013) 2429-2439.

[18] S. S. Sadiqqi, M. Iftikhar, Variational Iteration Method for the Solution of Seventh Order Boundary Value Problems using He's Polynomials, J. Assoc. Arab Univ. Basic Appl. Sci. 18 (2015) 60-65. doi.org/10.1016/j.jaubas.2014.03.001. 